\title{
Local macrophage proliferation, rather than recruitment from the blood, is a signature of Th2 inflammation*
}

\author{
Stephen J. Jenkins ${ }^{1}$, Dominik Ruckerl ${ }^{1}$, Peter C. Cook ${ }^{1}$, Lucy H. Jones ${ }^{1}$, Fred D. \\ Finkelman $^{2,5}$, Nico van Rooijen ${ }^{6}$, Andrew S. MacDonald ${ }^{1}$, and Judith E. Allen ${ }^{1, \#}$ \\ ${ }^{1}$ Centre for Immunity, Infection and Evolution, and the Institute for Immunology and Infection \\ Research, School of Biological Sciences, University of Edinburgh, Edinburgh, EH9 3JT, UK ${ }^{2}$ \\ Department of Medicine, Cincinnati Veterans Affairs Medical Center, Cincinnati, OH 45220, USA \\ ${ }^{3}$ Division of Immunology, Allergy, and Rheumatology, Department of Internal Medicine, \\ University of Cincinnati College of Medicine, Cincinnati, OH 45267, USA ${ }^{4}$ Division of \\ Immunobiology, Cincinnati Children's Hospital Medical Center, Cincinnati, OH 45229, USA 5 \\ Division of Rheumatology, Cincinnati Children's Hospital Medical Center, Cincinnati, OH 45229, \\ USA ${ }^{6}$ Department of Molecular and Cell Biology, Free University Medical Centre, Amsterdam, \\ The Netherlands
}

\section{Abstract}

\begin{abstract}
A defining feature of inflammation is the accumulation of innate immune cells in the tissue that are thought to be recruited from the blood. We reveal that a distinct process exists in which tissue macrophages undergo rapid in situ proliferation in order to increase population density. This inflammatory mechanism occurred during T helper 2 (Th2)-related pathologies under the control of the archetypal Th2 cytokine interleukin-4 (IL-4), and was a fundamental component of Th2 inflammation because exogenous IL-4 was sufficient to drive accumulation of tissue macrophages through self-renewal. Thus, expansion of innate cells necessary for pathogen control or wound repair can occur without recruitment of potentially tissue-destructive inflammatory cells.
\end{abstract}

\begin{abstract}
Recruitment of leukocytes from the blood is the key feature of inflammatory responses to tissue damage or infection. Prominent in this cascade is the rapid influx of granulocytes and monocytes that subsequently differentiate into inflammatory macrophages and/or dendritic cells (DC) $(1,2)$. This paradigm of classical 'type-1' inflammation is firmly grounded in processes elicited by microbial infection or necrotic cell death (2). Inflammation driven by helminth infection or allergy represents a distinct challenge to the immune system, and is characterized by the recruitment of cells that produce the cytokine IL-4, including eosinophils, basophils, and CD4+ Th2 cells $(3,4)$. Like classical inflammation, 'type-2' inflammation also results in the accumulation of large numbers of macrophages in the affected tissues, $(3,5-7)$, yet despite this IL- 4 and Th2 responses are often regarded as 'antiinflammatory'.
\end{abstract}

\footnotetext{
*This manuscript has been accepted for publication in Science. This version has not undergone final editing. Please refer to the complete version of record at http://www.sciencemag.org/. The manuscript may not be reproduced or used in any manner that does not fall within the fair use provisions of the Copyright Act without the prior, written permission of AAAS.

\#Address for correspondence: j.allen@ed.ac.uk.

Supporting Online Material www.sciencemag.org Material and Methods Figs. S1 to S10

35. Material and methods are available as supporting material on Science online.
} 
A key distinguishing feature of macrophages in type-2 inflammation is their polarization towards an alternative (also known as M2) state of activation, mainly driven by IL-4 and IL-13 and characterized by expression of a distinct repertoire of molecules including arginase I, resistin-like molecule alpha (RELMa) and Ym1/2 (8). It has been widely assumed $(1,8)$ that accumulation of alternatively activated macrophages occurs through a process of recruitment similar to macrophage influx during classical inflammation. Supporting this view, depletion of blood monocytes impairs recruitment of mucosal M2 macrophages during gastro-intestinal nematode infection (5). The intestinal environment presents unique challenges for the study of type-2-driven inflammation, however, because commensal bacteria may act as a classical trigger for monocyte influx.

\section{M2 macrophages accumulate independently of monocytes}

To examine type 2 inflammatory processes we profiled cell recruitment during infection with a nematode that resides within 'sterile' tissues. The rodent filarial nematode Litomosoides sigmodontis induces strongly Th2-biased responses (fig. S1A), and drives alternative macrophage activation in the pleural cavity (Fig. 1A), after migrating there from the skin (Fig. 1B). For comparison, intra-thoracic injection of thioglycollate was used as a benchmark of the classical inflammatory cascade (9), because when injected, it induces recruitment of macrophages to the pleural cavity. Indeed, thioglycollate injection resulted in the rapid influx of neutrophils (Gr- $\left.1^{\text {high }} / \mathrm{Ly}-6 \mathrm{G}^{\text {high }}\right)$ and $\mathrm{Gr}-1^{\text {intermediate (int) }} / \mathrm{Ly}-6 \mathrm{C}^{\text {high }}$ monocytes, leading to accumulation of large numbers of $\mathrm{F} 4 / 80^{+}$macrophages by day 3 (Fig. $1 \mathrm{C}$ and fig. S1B). In contrast, $L$. sigmodontis infection triggered very little neutrophil or Gr- $1^{\text {int }} / \mathrm{Ly}-6 \mathrm{C}^{\text {high }}$ monocyte recruitment to the pleural cavity during the first 15 days after infection (Fig. 1D and fig. S1C), even though larvae arrive at this site between 3 and 6 days post-infection (Fig. 1B). Despite this, large numbers of $\mathrm{F} 4 / 80^{+}$macrophages gradually accumulated in the cavity after day 6 post-infection (Fig. 1D), accompanied by their conversion to an alternatively activated phenotype (Fig. 1A). There was also a striking difference in surface phenotype of the macrophage populations elicited by nematode infection compared to classical inflammation: those elicited by infection expressed levels of F4/80 similar to resident F4/80 high macrophages from naïve tissues (fig. S1C), whereas those induced by thioglycollate expressed low F4/80 levels (fig. S1B), which is characteristic of macrophages recruited to the serous cavities under inflammatory conditions (10).

To determine the role of monocytes in macrophage accumulation, we injected clodronateloaded (CL) liposomes intravenously (i.v.). CL-liposomes block tissue infiltration by macrophages in a variety of inflammatory settings $(5,11,12)$. This procedure depletes both $\mathrm{Gr}-1^{\text {int }} / \mathrm{Ly}-6 \mathrm{C}^{\text {high }}$ monocytes, which are the precursors of $\mathrm{F} 4 / 80^{\text {low }}$ inflammatory macrophages recruited during classical inflammation, and $\mathrm{Gr}-1^{-} / \mathrm{Ly}-6 \mathrm{C}^{-}$monocytes, which have been proposed as precursors of both alternatively-activated and tissue resident macrophages $(1,13)$. Consistent with expectation, treatment with CL-liposomes blocked the accumulation of macrophages in the pleural cavity induced by thioglycollate injection (Fig. 1E). In contrast, administration of CL-liposomes during the period when macrophages accumulate in the cavity (Fig. 1F), or when worms first enter the cavity (fig. S2), had no effect on either the number of pleural macrophages at day 10 post-L. sigmodontis infection or the frequency of those expressing alternative activation markers. Macrophage accumulation occurred despite almost complete removal of $\mathrm{Gr}-1^{-} / \mathrm{Ly}-6 \mathrm{C}^{-}$monocytes from the blood throughout the entire period after CL-liposome treatment and transient depletion of Gr-1 $1^{\text {int }} /$ Ly-6C ${ }^{\text {high }}$ monocytes (Fig. 1G). 


\section{Macrophages proliferate in situ}

These data raised the possibility that the large increase in macrophages seen during infection was the result of expansion of the resident population rather than blood cell recruitment. Whilst terminal differentiation of most mammalian cells typically results in reduced proliferative capacity, there is evidence that cells of the mononuclear phagocyte system can self-replicate in the periphery during development (14) or to maintain the tissue resident population $(15,16)$, but not as a classical inflammatory mechanism. Accordingly, on average $17 \%$ of resident pleural macrophages in naïve mice were positive for Ki67, a marker of proliferation expressed throughout cell-cycle, and $1 \%$ were in S-phase, as measured by a $3 \mathrm{hr}$ pulse of BrdU (Fig. 2A\&B and fig. S3A). In contrast, less than $2 \%$ of thioglycollateelicited macrophages expressed Ki67 and only $0.2 \%$ were in S-phase (Fig. 2A). Remarkably, pleural macrophages isolated ten days after infection with L. sigmodontis exhibited a substantial increase in the frequency of dividing cells. On average $38 \%$ of cells in the pleural cavity were positive for Ki67 and 6\% were in S-phase (Fig. 2B). BrdU ${ }^{+}$ macrophages seen in the pleural cavity were not recruited from the blood because no blood monocytes incorporated BrdU in this 3 hour period (fig. S3B).

To determine whether macrophage proliferation occurs in a distinct type- 2 setting, we looked in the peritoneal cavity following surgical implantation of the human parasitic filarial nematode Brugia malayi. Incision and suture of the abdominal wall without parasite implantation was used to control for the surgical procedure and provides a model of incisional wounding that is characterized by IL-4Ra-dependent alternative macrophage activation (17). Both implantation of $B$. malayi and surgery alone resulted in an increased frequency of $\mathrm{BrdU}^{+}$and $\mathrm{Ki}^{+} 7^{+}$peritoneal macrophages, although this was only maintained following parasite infection (fig. S4), when Th2 cells become activated (17). Ki67 macrophages have also been observed in the lungs of hookworm infected mice (7) and epidermal langerhans cells up-regulate proliferation during atopic dermatitis (14) supporting the notion that macrophage proliferation is a characteristic common to various type-2 inflammatory settings.

\section{IL-4 instructs proliferative expansion}

IL-4 is important for macrophage accumulation during numerous helminth infections $(5,6)$. We thus infected IL-4-deficient mice with $L$. sigmodontis to test the requirement for IL-4 in proliferative expansion of tissue macrophages. Significantly fewer macrophages from IL-4deficient mice were capable of proliferating, as determined by BrdU incorporation, leading to a failure of these cells to accumulate in the pleural cavity (Fig. 2C). As expected, macrophages that were present in IL-4-deficient mice were also unable to M2 activate, whereas worm burdens were indistinguishable between strains at this early stage of infection, as previously described (18). To demonstrate that IL-4 drives macrophage proliferation in vivo, mice were injected via the intraperitoneal (i.p.) route with recombinant IL-4 complexed to IL-4 antibody (IL-4c), which increases the bioactive half-life of the cytokine (19). Delivery of IL-4c to naïve mice led to an elevation in peritoneal cellularity over a 4 day period that was due primarily to increased macrophage numbers, all of which exhibited an alternative activation phenotype (Fig. 3A). IL-4-elicited macrophages expressed very high levels of F4/80 (mean fluorescence intensity (MFI) of 7300 \pm 680 versus $4900 \pm 380$ in PBS-treated controls; mean \pm SEM) and neither inflammatory monocytes nor neutrophils were recruited (fig. S5), consistent with a process distinct from classical inflammation $(2,10)$. Critically, the rate of macrophage proliferation was increased compared to mice receiving PBS alone (Fig. 3A), whereas no proliferation or accumulation was observed in IL-4Ra-deficient mice (fig. S6). Thus, delivery of exogenous IL-4 was able 
to mimic the induction of proliferation and accumulation of macrophages observed during type-2 infection.

Macrophage proliferation in response to IL-4c was not restricted to the peritoneal cavity. There was an increase in number and frequency of Kupffer cells in the liver (Fig. 3B) as previously shown (19), and the number of macrophages in the pleural cavity (Fig. 3C) and both of these populations exhibited increased incorporation of BrdU (Fig. 3, B and C). Thus, IL-4 can induce tissue macrophage proliferation in diverse body tissues and we propose that in an environment with high IL-4 the tissue macrophage population will undergo extensive proliferation.

\section{Self-renewal of resident tissue macrophages}

To establish definitively whether IL-4-driven accumulation of tissue macrophages is due to enhanced turnover of the resident population, we utilized a modified technique for creating bone marrow chimeric animals (20). Engraftment of bone marrow cells into lethally irradiated mice results in the replacement of most resident peritoneal macrophages by cells from transplanted bone marrow $(16,20)$. In the modified protocol, the upper body of $C d 45.1$ congenic mice was shielded during irradiation to protect resident pleural macrophages from harmful effects of direct exposure while allowing engraftment of donor CD45.2 $2^{+}$bone marrow. Mice were reconstituted for 8 weeks before i.p. injection of IL-4c or PBS, or intrathoracic injection of thioglycollate. As expected, partial shielding led to a mixed chimerism in peripheral blood monocytes (Fig. 3D) and granulocytes (fig. S7) in all groups of mice, with approximately $25 \%$ to $30 \%$ of donor origin. In contrast, only $3 \%$ of pleural macrophages were of donor origin in PBS-injected mice (Fig. 3D), which demonstrated that little replacement of these cells occurred from the bone-marrow under steady-state conditions. Despite a 3-fold increase in cell number, the same low-level chimerism (approximately $3 \%$ donor-derived cells) was observed in pleural cavity macrophages of mice treated with IL-4c (Fig. 3D). Identical data were obtained for pleural macrophages from L. sigmodontis-infected chimeric mice (fig. S8). In contrast, macrophages elicited by injection of thioglycollate exhibited a high frequency of chimerism indistinguishable from that observed in blood monocytes and consistent with their differentiation from newly recruited cells of bone-marrow origin (Fig. 3D). These data reveal that IL-4 and filarial nematode infection elicit macrophages by expansion of the tissue resident population, independent of recruitment of bone marrow-derived precursor cells.

Type- 1 and type- 2 immunity represent ancient innate pathways with fundamentally different purposes; one controls microbial pathogens (21) and the other macroparasites (22). Profound differences in amino acid utililization (23) and energy metabolism (24) of polarized macrophage populations illustrates the broad divergence of these pathways. Similarly, the IL-4-driven process of proliferative expansion described here contrasts sharply with the well-defined classical inflammatory process of recruitment (2) that predominates during type-1 infections (21). The capacity for IL-4-driven proliferation to achieve similar cell numbers to classical cell recruitment (Fig. 3C) suggests that this is a fundamental alternative inflammatory pathway. To verify that rapid proliferative expansion of macrophages represents an innate mechanism of inflammation, we injected RAG-1-deficient mice with IL-4c. Just as thioglycollate induced macrophage recruitment occurs normally in RAG-1deficient mice (17), IL-4c-mediated proliferative expansion was unaltered (Fig. 3E). These data reveal that accelerated macrophage turnover is an innate response to IL-4, analogous to the classical inflammatory response to pro-inflammatory cytokines. In natural settings, the source of IL-4 will depend upon the location and stage of type- 2 inflammation, and can be innate IL-4 producing cells, or adaptive Th2 cells, as illustrated previously using the surgical wounding or B. malayi peritoneal implant models, respectively (17). 


\section{Proliferation of recruited tissue macrophages}

Classical inflammatory stimuli such as microbial insult frequently co-localize with IL-4 production. To assess the impact of IL-4 in a setting where macrophages are actively recruited by classical stimuli, we simultaneously injected thioglycollate and IL-4c into the peritoneal cavity. Injection of thioglycollate in the presence of IL-4 induced the accumulation of macrophages expressing low levels of F4/80 (fig. S9), indicative of a recruited monocyte-derived population (10). In a separate experiment, proliferating M2 macrophages elicited by intra-pleural injection of thioglycollate in combination with IL-4c i.p. were confirmed to be of blood origin using the modified bone marrow chimeric animals described earlier (fig. S10)(20). Critically, thioglycollate-elicited monocyte-derived macrophages had the capacity to proliferate and become alternatively activated following treatment with IL-4 (Fig. 4 and fig. S10). Such a situation of mixed recruitment/expansion is likely to apply during infection with gastro-intestinal nematodes where, despite evidence of recruitment from the blood, the total number of macrophages that accumulate in the gut mucosa is far reduced in IL-4-deficient mice (5). Collectively, our data demonstrate that both resident and recruited macrophages can alternatively activate and be driven to proliferate by a Th2 environment in vivo. Thus, there is neither a specific precursor for M2 macrophages nor is proliferative capacity restricted by lineage.

\section{Two modes of inflammation}

The obvious benefit of classical inflammation is the rapidity with which large numbers of macrophages can accumulate in the tissues but the maintenance of a circulating precursor pool throughout the body is energetically costly. In contrast, the slower mechanism of local expansion in type-2 inflammation does not require enhanced bone marrow activity (Fig. 3D) and may deliberately avoid inflammatory cell recruitment and the associated potential for tissue damage. Indeed, IL-4 can actively block granulocyte recruitment during delayed-type hypersensitivity (25) and instruct macrophages to down-regulate production of proinflammatory chemotactic factors (26). This would be of particular benefit during wound healing, for example, where type 2-activated macrophages accelerate repair $(22,27,28)$ and sustained classical inflammation results in a failure to heal $(29,30)$. Thus, we propose that proliferation at site is an alternative mechanism of inflammation that allows macrophages to accumulate in sufficient numbers to perform critical functions such as parasite sequestration or wound repair in the absence of potentially damaging cell recruitment. This has broad therapeutic implications in settings where Th2-driven macrophages have key positive or negative function, such as in wound healing, autoimmunity or the tumor micro-environment.

\section{Summary}

We have discovered that two distinct innate processes exist through-which macrophages colonize tissues during inflammation; recruitment of monocytes controlled by classical inflammatory stimuli and rapid proliferation of the tissue resident population that is orchestrated by IL-4 and occurs independently of recruitment from the hematopoietic pool.

\section{Supplementary Material}

Refer to Web version on PubMed Central for supplementary material.

\section{Acknowledgments}

We thank Professors R. Zamoyska, F. Brombacher and D. Gray for critical reading of the manuscript, and A. Fulton and S. Duncan for expert technical assistance. This study was funded by the MRC UK (G0600818 to JEA), with 
additional support from the EU ("SCOOTT"; INCO-CT-2006-03232), MRC UK (G0701437 to ASM) the US Department of Veterans Affairs (Merit Award to FDF) and the National Institutes of Health (RO1 AI070300 to FDF). LHJ was funded by a Wellcome Trust PhD studentship.

\section{References and Notes}

1. Auffray C, Sieweke MH, Geissmann F. Annu Rev Immunol. 2009; 27:669. [PubMed: 19132917]

2. Soehnlein O, Lindbom L. Nat Rev Immunol. 2010; 10:427. [PubMed: 20498669]

3. Anthony RM, Rutitzky LI, Urban JF Jr. Stadecker MJ, Gause WC. Nat Rev Immunol. 2007; 7:975. [PubMed: 18007680]

4. Voehringer D, Shinkai K, Locksley RM. Immunity. 2004; 20:267. [PubMed: 15030771]

5. Anthony RM, et al. Nat Med. 2006; 12:955. [PubMed: 16892038]

6. MacDonald AS, Loke P, Martynoga R, Dransfield I, Allen JE. Med Microbiol Immunol. 2003; 192:33. [PubMed: 12592561]

7. Siracusa MC, Reece JJ, Urban JF Jr. Scott AL. J Leukoc Biol. 2008; 84:1422. [PubMed: 18719016]

8. Gordon S, Martinez FO. Immunity. 2010; 32:593. [PubMed: 20510870]

9. Kuziel WA, et al. Proc Natl Acad Sci U S A. 1997; 94:12053. [PubMed: 9342361]

10. Ghosn EE, et al. Proc Natl Acad Sci U S A. 2010; 107:2568. [PubMed: 20133793]

11. Getts DR, et al. J Exp Med. 2008; 205:2319. [PubMed: 18779347]

12. Smith P, et al. J Immunol. 2007; 178:4557. [PubMed: 17372014]

13. Sunderkotter C, et al. J Immunol. 2004; 172:4410. [PubMed: 15034056]

14. Chorro L, et al. J Exp Med. 2009; 206:3089. [PubMed: 19995948]

15. Daems WT, de Bakker JM. Immunobiology. 1982; 161:204. [PubMed: 7047370]

16. Merad M, et al. Nat Immunol. 2002; 3:1135. [PubMed: 12415265]

17. Loke P, et al. J Immunol. 2007; 179:3926. [PubMed: 17785830]

18. Le Goff L, Lamb TJ, Graham AL, Harcus Y, Allen JE. Int J Parasitol. 2002; 32:1277. [PubMed: 12204227]

19. Milner JD, et al. Blood. 2010; 116:2476. [PubMed: 20570861]

20. Murphy J, Summer R, Wilson AA, Kotton DN, Fine A. Am J Respir Cell Mol Biol. 2008; 38:380. [PubMed: 18192503]

21. Serbina NV, Jia T, Hohl TM, Pamer EG. Annu Rev Immunol. 2008; 26:421. [PubMed: 18303997]

22. Allen JE, Wynn TA. PLoS Pathog. 2011; 7:e1002003. [PubMed: 21589896]

23. Munder M, Eichmann K, Modolell M. J Immunol. 1998; 160:5347. [PubMed: 9605134]

24. Vats D, et al. Cell Metab. 2006; 4:13. [PubMed: 16814729]

25. Fine JS, et al. Inflammation. 2003; 27:161. [PubMed: 14527170]

26. Loke P, et al. BMC Immunol. 2002; 3:7. [PubMed: 12098359]

27. Lucas T, et al. J Immunol. 2010; 184:3964. [PubMed: 20176743]

28. Ruffell D, et al. Proc Natl Acad Sci U S A. 2009; 106:17475. [PubMed: 19805133]

29. Ashcroft GS, et al. J Clin Invest. 2003; 111:1309. [PubMed: 12727922]

30. Eming SA, Krieg T, Davidson JM. J Invest Dermatol. 2007; 127:514. [PubMed: 17299434]

31. Herbert DR, et al. Immunity. 2004; 20:623. [PubMed: 15142530]

32. Finkelman FD, et al. J Immunol. 1993; 151:1235. [PubMed: 8393043]

33. Phythian-Adams AT, et al. J Exp Med. 2010; 207:2089. [PubMed: 20819926]

34. Van Rooijen N, Sanders A. J Immunol Methods. 1994; 174:83. [PubMed: 8083541] 
A

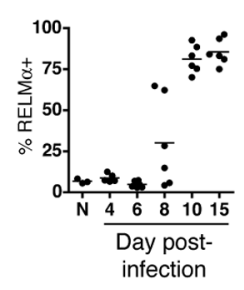

C

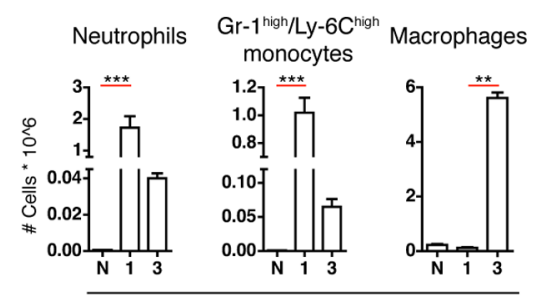

Day post-injection

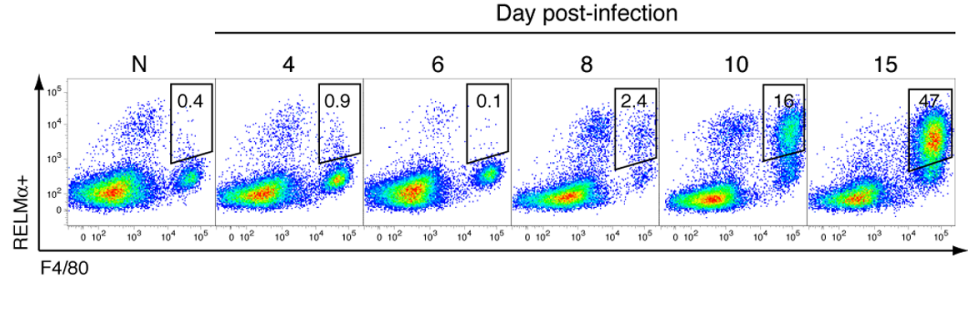

B

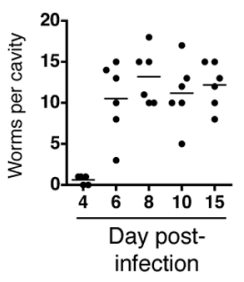

E

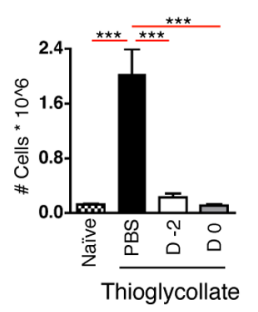

$\mathbf{F}$

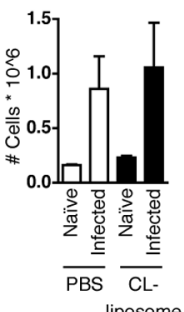

D

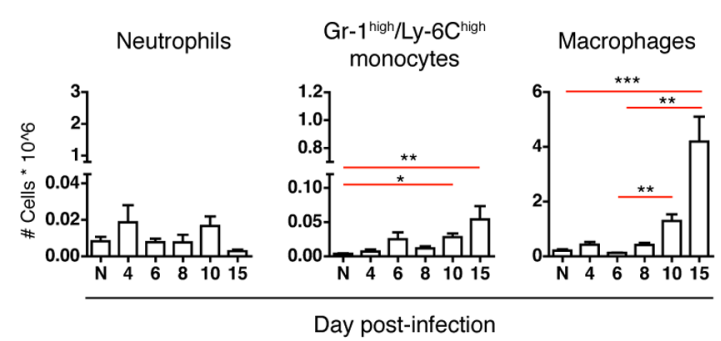

Fig. 1.

Alternatively activated macrophages accumulate independently of blood monocytes. (A) Proportion of pleural cavity macrophages staining positive for RELMa following infection with $L$. sigmodontis and representative flow cytograms gated on $\mathrm{CD}_{11} 1 \mathrm{~b}^{+}$Siglec- $\mathrm{F}^{-}$cells for clarity. N = naïve. (B) Pleural cavity worm burden from mice in (A). (C) Pleural cavity cells elicited upon intra-thoracic injection of thioglycollate or (D), after infection with $L$. sigmodontis, using flow cytometric gating schemes shown in fig. $\mathrm{S} 1, \mathrm{D}$ and $\mathrm{E} . \mathrm{N}=$ naïve. (E) Total pleural macrophages elicited by thioglycollate after i.v. injection of PBS, or CLliposomes from 2 days prior (D -2) or on the day of (D 0) thioglycollate injection. (F) Total pleural macrophages and the proportion producing RELMa on day 10 post-L. sigmodontis infection after daily i.v injection of CL-liposomes or PBS from days 6-9. (G) Frequency of Gr-1-/Ly-6C ${ }^{-}$CD115 ${ }^{+}$(B1) or Gr- $1^{\text {int } / L y-6 C^{\text {high }}}$ CD115+ (B2) monocytes in CD11b blood leukocytes on day 3 and 10 post- $L$. sigmodontis infection after daily i.v injection of PBS or CL-liposomes from days 2-5. Values in italics represent the mean \pm SEM of 8 mice. (A-G) All results are representative of 2-3 independent experiments. Graphs present mean \pm SEM of 3-8 mice. (C and D) $* P<0.05, * * P<0.01$ and $* * * P<0.001$, as determined using KruskalWallis test, with Dunn's post-test comparing all groups. (E) $* * * P<0.001$, as determined using one-way analysis of variance (ANOVA) with Tukey's post-test for multiple comparisons. 
A
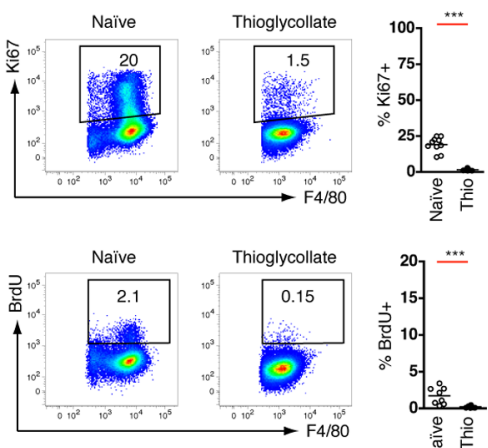

B
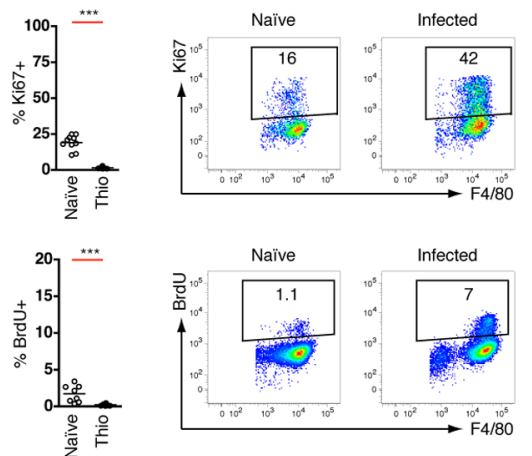

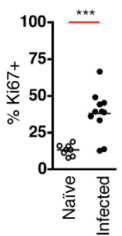

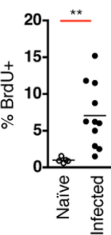

C

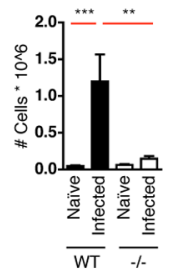

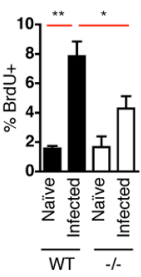

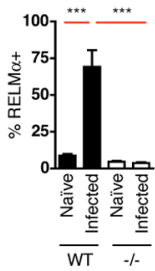

Fig. 2.

Type-2 inflammation drives in situ proliferation of tissue macrophages. (A) Flow-cytometric analysis of Ki67 expression or BrdU incorporation by pleural macrophages from naïve mice or 3 days post-thioglycollate (Thio) injection. BrdU was administered 3 hours prior to analysis. Gating schemes for Ki67 and BrdU analysis are shown in fig, S1B and 3A, respectively. Results are pooled data from 2 independent experiments with between 8 and 11 total mice per group. (B) As (A) but BrdU injection and analysis of cells was performed on day 10 post-infection with $L$. sigmodontis. Results are pooled data from 2 independent experiments with between 5 and 11 total mice per group, and are representative of at least 3 further independent experiments. (C) Total pleural macrophages and the proportion staining positive for BrdU and RELMa from naïve and day 10 L. sigmodontis-infected C57BL/6 (WT) and $I 14^{-1-}(-/-)$ mice, as determined by flow cytometry. Data are representative of two independent experiments. Graphs present mean \pm SEM of 7-8 mice per group. (A and B) $* * P<0.01$ and $* * * P<0.001$, as determined by 2 -tailed $t$ test. (C) $* P<0.05$, $* * P<0.01$ and $* * * P<0.001$, as determined using one-way ANOVA with Tukey's post-test for multiple comparisons. 
A
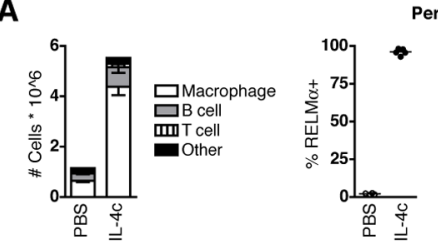

Peritoneal macrophages
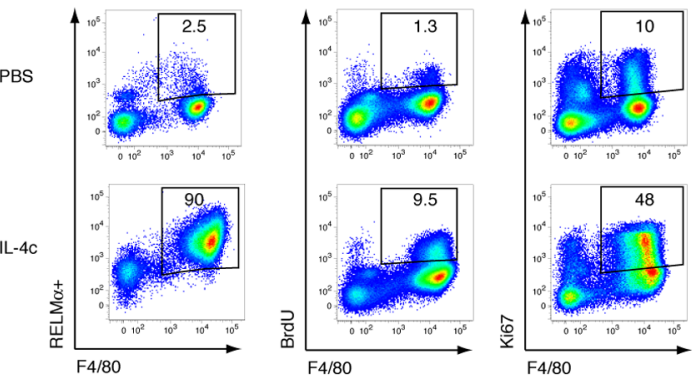

B
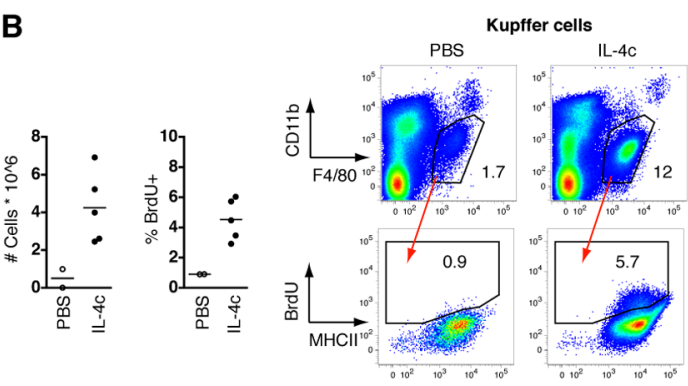

C
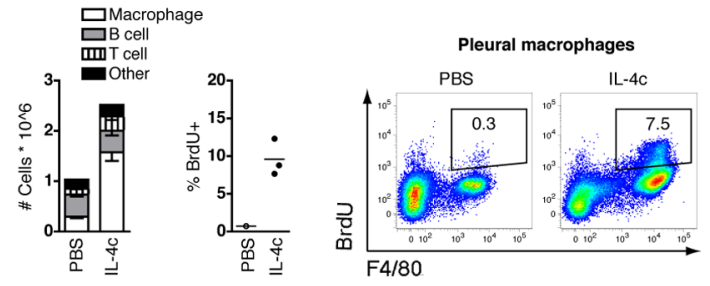

D
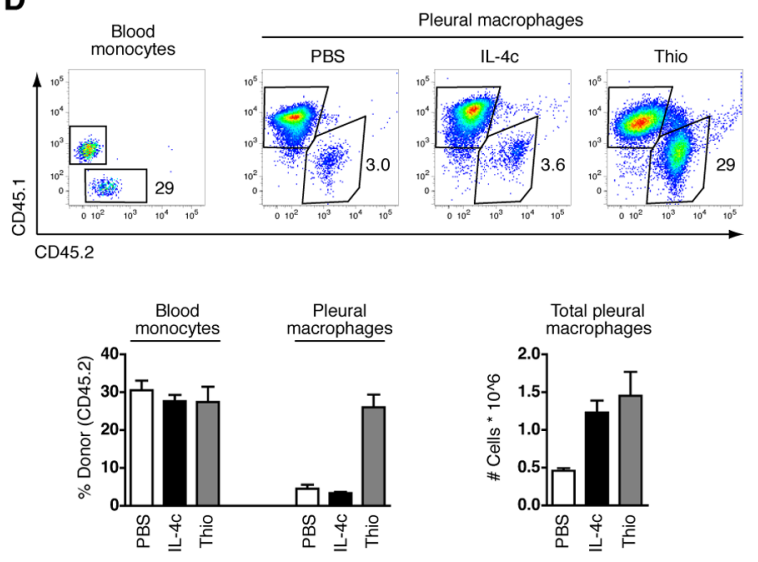

E

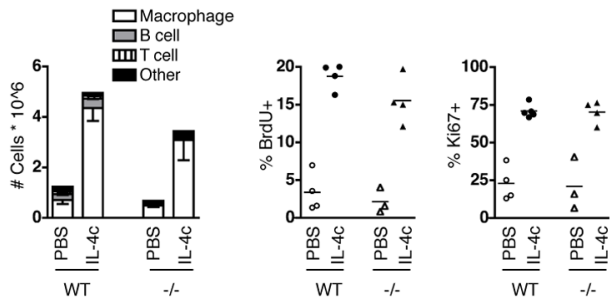

Fig. 3.

Macrophage proliferation is an innate tissue hyperplasia triggered by IL-4. Mice were treated i.p. with PBS or IL-4c on days 0 and 2 and analyzed on day 4. (A) Composition of peritoneal cells, the proportion of peritoneal macrophages positive for BrdU, Ki67 or RELMa, and representative flow cytograms depicting all peritoneal cells. Graphs present mean \pm SEM of 5 mice per group; representative of 10 independent experiments. (B) Total $\mathrm{F} 4 / 80^{+} \mathrm{CD}_{11 b^{\text {low }}}$ Kupffer cells, BrdU incorporation by Kupffer cells, and gating scheme depicting Kupffer cells and BrdU versus MHCII staining as determined by flow cytometry. Data shown are of livers pooled from 3 PBS-treated mice, or individual IL-4c-treated mice, and are combined from two independent experiments. (C) As (A) but in the pleural cavity. Graph presents mean \pm SEM of 3 mice per group for cell composition, whereas BrdU incorporation is of pooled cells from 3 PBS-treated mice, or individual IL-4c-treated mice. Data are representative of 5 independent experiments. (D) Flow cytometric analysis of chimerism of blood monocytes and pleural macrophages and number of pleural macrophages in $C d 45.1$ congenic mice given CD45.2 ${ }^{+}$bone marrow cells after partial-body irradiation, and subsequently treated with PBS or IL-4c i.p., or intra-thoracic thioglycollate at week 8 post reconstitution. Representative CD45.1 and CD45.2 staining gated on CD11b ${ }^{+}$ 
$\mathrm{CD} 115^{\text {high }}$ blood monocytes or $\mathrm{F} 4 / 80^{+}$pleural macrophages. Graphs depict mean \pm SEM of 4 mice per group; representative of 2 independent experiments. (E) As (A) but in C57BL/6 (WT) and $\operatorname{Rag}^{-{ }^{--}}(-/-)$mice. Graphs depict mean \pm SEM of 3-4 mice per group; representative of 2 independent experiments. 

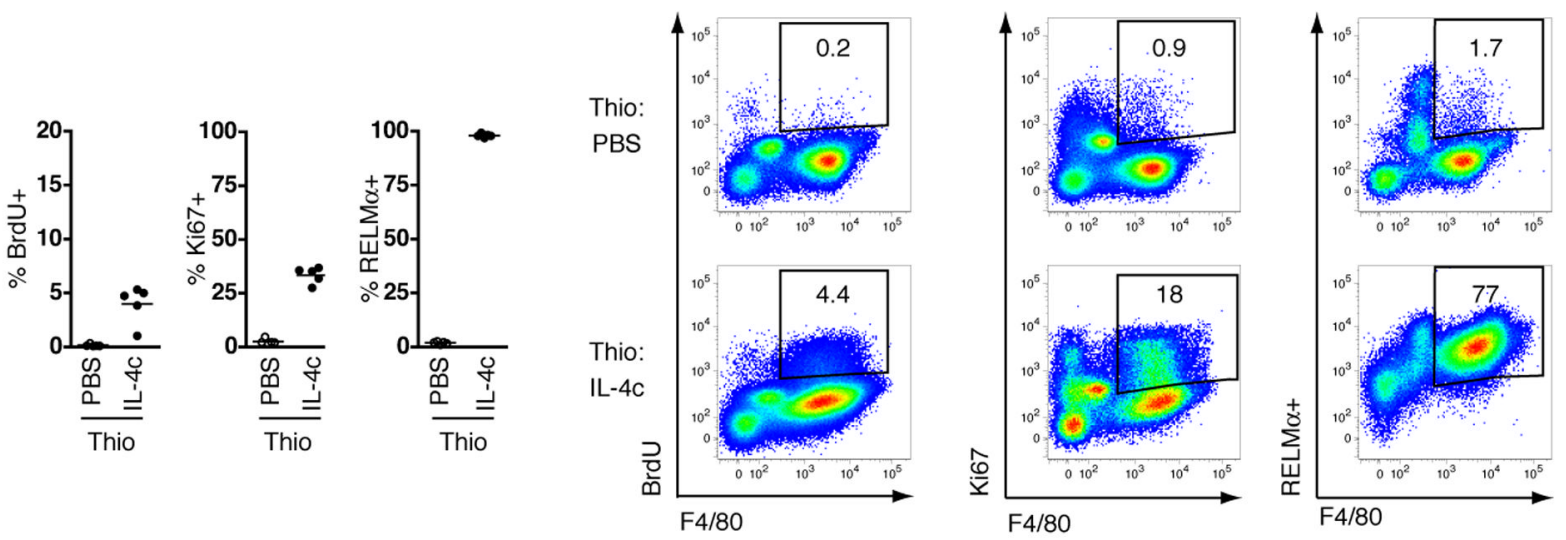

Fig. 4.

IL-4 triggers inflammatory macrophages to proliferate and alternatively activate. The proportion of peritoneal macrophages positive for BrdU, Ki67 or RELMa are shown, and representative flow cytograms depicting all peritoneal cells, from mice injected i.p. simultaneously with thioglycollate and PBS or IL-4c. IL-4c and PBS injections repeated on day 2 and analysis performed on day 4 . Data are representative of two independent experiments, with 5 mice per group. 